\title{
Stadienspezifische Anpassung \\ Diagnostik und Therapie bei akutem Koronarsyndrom
}

\author{
H.-P. Hermann, Herzzentrum Göttingen, Abteilung Kardiologie und Pneumologie, Georg-August-Universität Göttingen \\ (Direktor: Prof. Dr. G. Hasenfuß)
}

NOTFALLMEDIZIN 2003; 29: 462-467

Nach der neuen Definition der WHO liegt stets dann ein Myokardinfarkt vor, wenn bei instabiler Angina pectoris biochemische Zeichen/Marker der Myokardschädigung nachweisbar sind, also erhöhte CK-MB oder kardiale Troponin-Werte (Troponin T oder I) gemessen werden. Aus der Kenntnis der gemeinsamen Pathophysiologie der akuten Koronarsyndrome leitet sich die aktuelle Therapie ab, die stadienspezifisch angepasst wird. Aus großen epidemiologischen Erhebungen ist bekannt, dass nicht nur die Prognose des ST-Elevationsinfarkts sondern auch die der akuten Koronarsyndrome ohne ST-Elevation ungünstig ist. Dies begründet ein aggressives therapeutisches Vorgehen analog zum akuten Myokardinfarkt mit ST-Hebung.

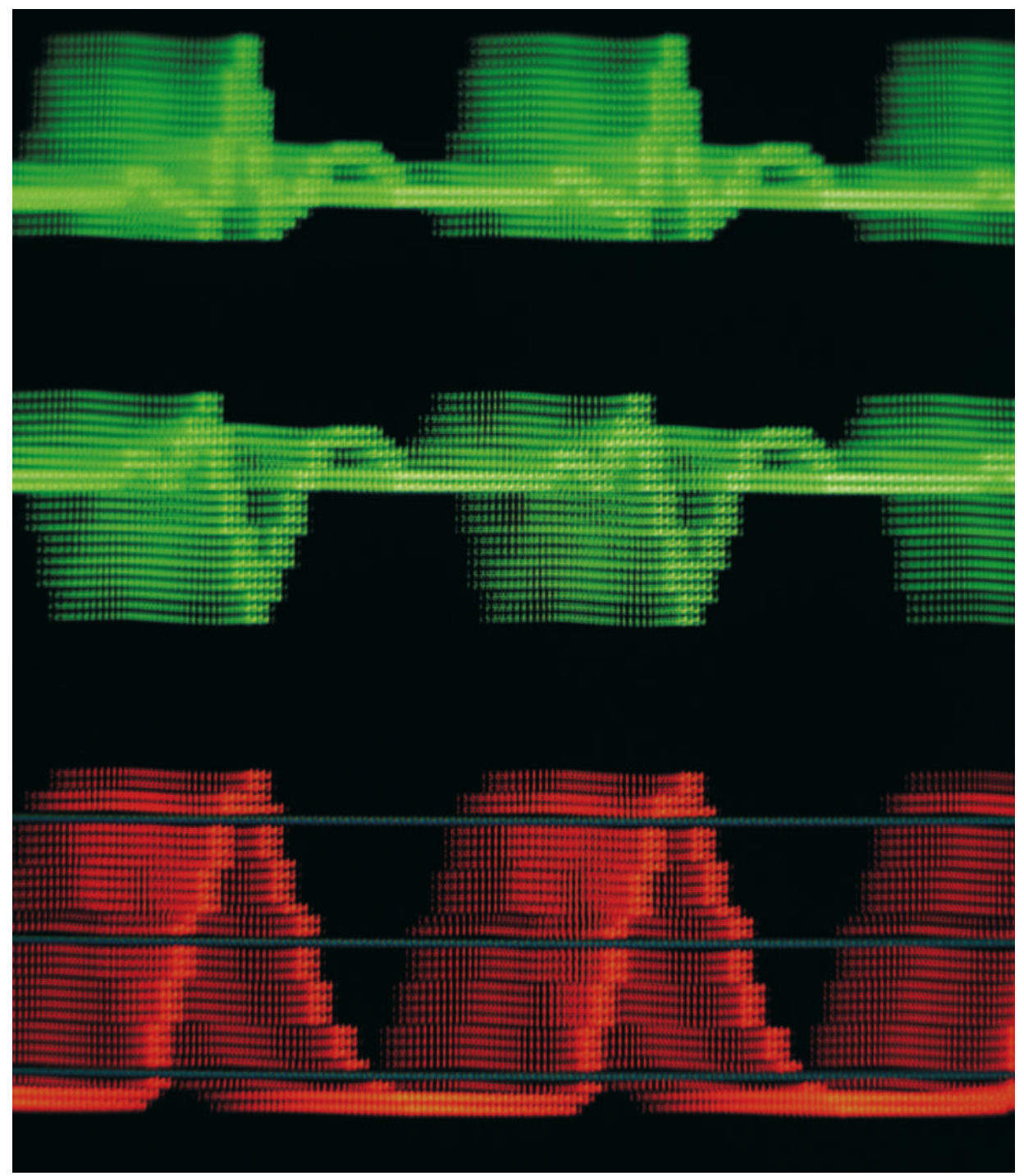

$\mathrm{D}$ er Begriff „Akutes Koronarsyndrom" umfasst drei differente klinische Entitäten:

1. instabile Angina pectoris (iAP),

2. den nicht-transmuralen Myokardinfarkt beziehungsweise non-ST-Elevations-Myokardinfarkt (NSTEMI) sowie

3. den transmuralen Myokardinfarkt beziehungsweise ST-Elevations-Myokardinfarkt (STEMI) (Abb. 1).

Allen drei Entitäten liegt pathophysiologisch eine gemeinsame Ursache zugrunde: erodierte und/ oder rupturierte atherosklerotische Plaques der Koronararterien. Die unterschiedlichen klinischen Ausprägungsformen ergeben sich durch das Ausmaß der Anlagerung von Thromben und der resultierenden distalen Embolisierung. So führt eine komplette Gefäßokklusion ohne suffiziente Kollateralversorgung zum Bild des ST-Elevationsinfarkts, ein partieller Verschluss mit signifikanter distaler Embolisation führt zum Bild des non-ST-Elevationsinfarkts, oder bei geringem Ausmaß der peripheren Embolisation zur instabilen Angina pectoris. Nach der neuen Definition der WHO liegt stets dann ein Myokardinfarkt vor, wenn bei instabiler Angina pectoris biochemische Zeichen/Marker der Myokardschädigung nachweisbar sind, also erhöhte CK-MB oder kardiale Troponin-Werte (Troponin T oder I) gemessen werden.

Aus der Kenntnis der gemeinsamen Pathophysiologie der akuten Koronarsyndrome leitet sich die aktuelle Therapie ab, die Stadienspezifisch angepasst wird. Aus großen epidemiologischen Erhebungen ist bekannt, dass nicht nur die 
Prognose des ST-Elevationsinfarkts sondern auch die der akuten Koronarsyndrome ohne ST-Elevation ungünstig ist: im EuroHeartSurvey 2001-2002 in 25 europäischen Ländern wurde eine 6-Monats-Mortalität von $12 \%$ für NSTEMI und instabile Angina pectoris festgestellt. Dies begründet ein aggressives therapeutisches Vorgehen analog zum akuten Myokardinfarkt mit ST-Hebung.

\section{Initiale Diagnostik}

Patienten mit typischer Angina pectoris-Symptomatik $\geq 20$ Minuten Dauer müssen nach kurzer Erhebung der Anamnese rasch körperlich untersucht werden, um nicht-myokardiale Ursachen der Beschwerden - zum Beispiel Pneumothorax, Pleuritis, Perikarditis, Aortenklappenstenose, Aortendissektion, Cholecystitis - zu erkennen. Anschließend wird unverzüglich ein 12-Kanal-EKG abgeleitet (Abb. 2). Finden sich hier monophasische ST-Streckenhebungen in zwei benachbarten Ableitungen $(\geq 0,1 \mathrm{mV}$ in den Extremitätenableitungen oder $\geq 0,2 \mathrm{mV}$ in den Brustwandableitungen) oder ist ein kompletter Linksschenkelblock vorhanden, welcher nicht vorbekannt ist, so ist von einem transmuralen Myokardinfarkt (STEMI) auszugehen und die entsprechende Therapie sofort einzuleiten. Sind weder STHebungen, noch ein neu aufgetretener LSB vorhanden, liegt zunächst kein transmuraler Infarkt vor. Zur Unterscheidung zwischen instabiler Angina pectoris (iAP) und non-STElevations-Myokardinfarkt (NSTEMI) dient jetzt die Bestimmung biochemischer Marker der Myokardschädigung, das heißt in der Regel der Nachweis erhöhter kardialer Troponinspiegel (Tab. 1). Bei negativen Befunden und Verdacht auf nichtmyokardiale Genese der Symptomatik können jetzt auch ergänzende diagnostische Verfahren zur weiteren Abklärung eingeleitet werden.

\section{Risikobeurteilung für Patien- ten mit akutem Koronar- syndrom ohne ST-Elevation (iAP und NSTEMI)}

Die Behandlungsstrategie hängt bei dieser heterogenen Patientengruppe davon ab, wie hoch das
Risiko für die Progression zu einem transmuralen Myokardinfarkt (STEMI) oder zum Tod des Patienten eingeschätzt wird. Folgende Parameter werden zur Risikoeinschätzung für den individuellen Patienten herangezogen:

- Vorhandene Risikofaktoren einer koronaren Herzkrankheit

- Klinisches Bild (z.B. Intensität und Frequenz der AP-Episoden)

- Elektrokardiogramm (dynamische EKG-Veränderungen)

- Marker einer myokardialen Schädigung (Troponine)

- (Marker der Entzündungsaktivität (hsCRP, BNP))

- Echokardiogramm (regionale Wandbewegungsstörungen)

- Belastungstest vor Entlassung (nach Abklingen der akuten Symptomatik)

- Koronarangiographie (akut oder elektiv)

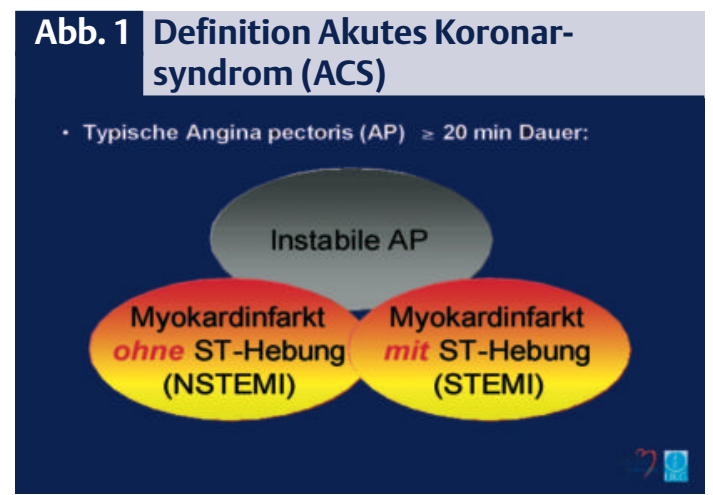

Aufgrund aktueller Studiendaten ist eine Risikostratifizierung in zwei Gruppen etabliert und klinisch validiert (Abb. 3).

\section{Hohes Risiko für Progression zum transmuralen Infarkt}

Ein hohes Risiko für eine Progression zum transmuralen Infarkt haben Patienten mit rezidivierender

\section{Tab. 1 Empfehlungen der Europäischen Gesellschaft für Kardio- logie bei Verdacht auf akute ischämische Herzkrankheit}

- Ableiten eines Ruhe-EKG's und Beginn eines kontinuierlichen MehrkanalMonitorings der ST-Strecke (oder engmaschige wiederholte EKG-Ableitungen, wenn eine Monitorüberwachung nicht möglich ist).

- Bestimmung von Troponin T oder I bei Aufnahme sowie - bei Werten im Normalbereich - erneute Bestimmung nach 6-12 Stunden.

- Bestimmung von Myoglobin und/oder CK-MB bei Patienten mit frischen (<6 Stunden) Symptomen als frühe Marker eines Myokardinfarkts sowie bei Patienten mit erneuter Ischämie nach frischem Infarkt ( $<2$ Wochen) zum Ausschluss eines Re-Infarkts.

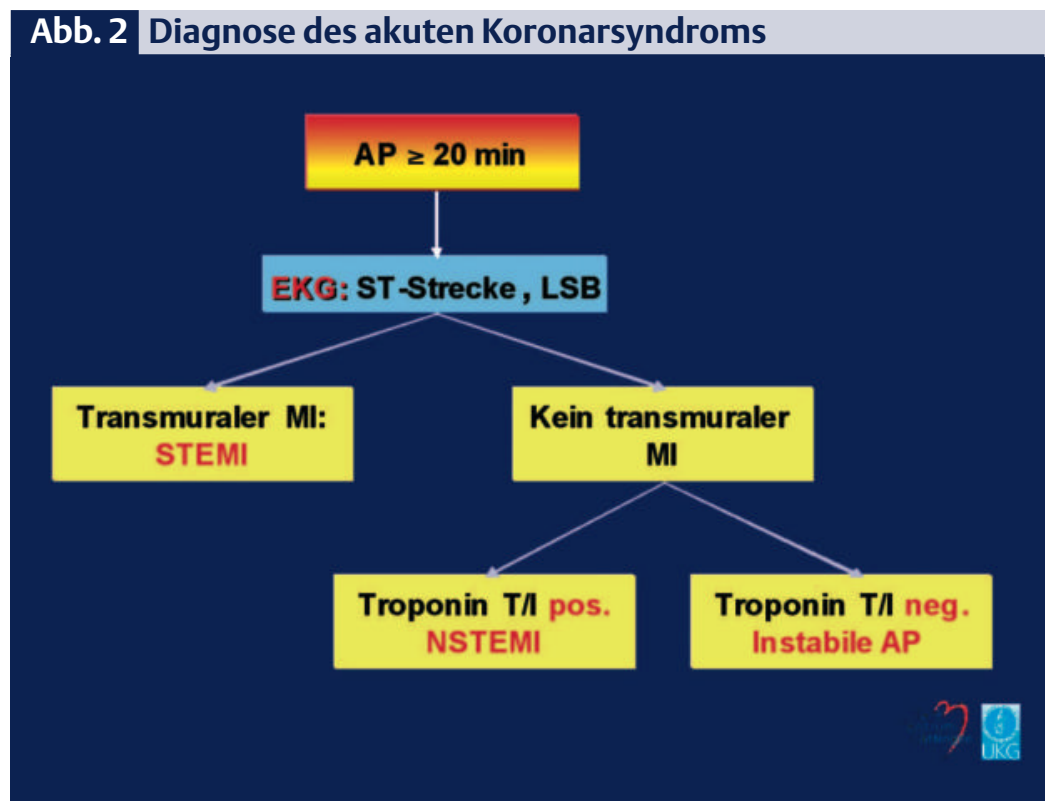




\section{Abb. 3 Risikostratifizierung in der akuten Phase}

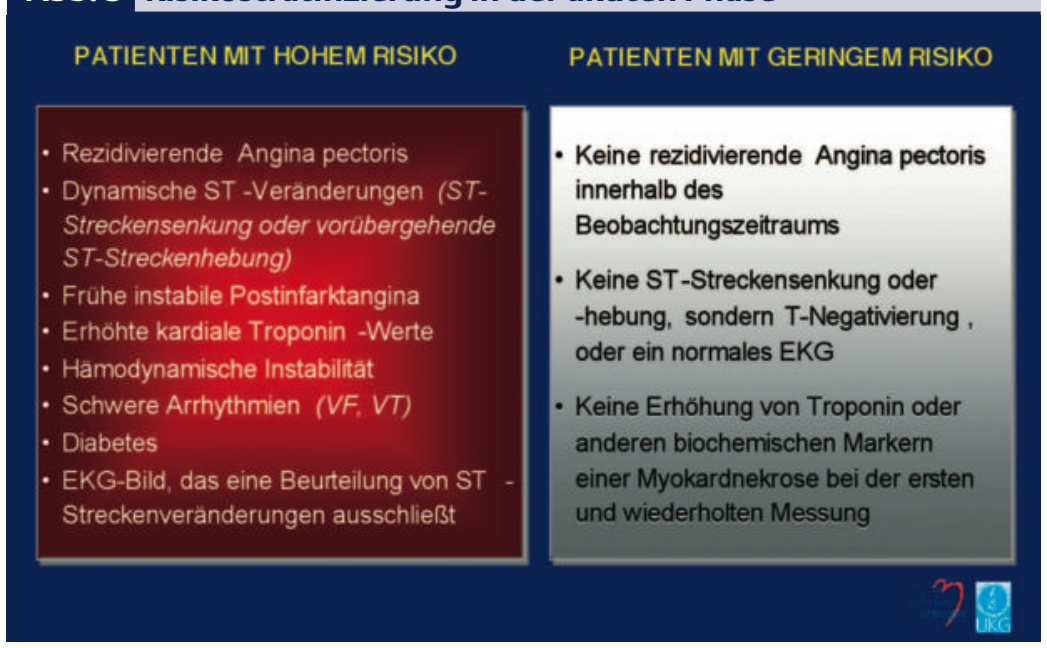

Angina pectoris im Beobachtungszeitraum, dynamischen ST-Streckenveränderungen mit ST-Senkungen oder passageren ST-Hebungen, Patienten mit früher PostinfarktAngina pectoris, erhöhten kardialen Troponin-Werten, hämodynami- scher Instabilität, Rhythmusinstabilität mit Episoden von Kammerflimmern oder anhaltenden Kammertachykardien. Zur Risikogruppe gehören zudem Patienten mit Diabetes mellitus und Patienten mit EKGBefunden, die eine Beurteilung von ST-Streckenveränderungen ausschließen (vorbestehende Schenkelblockbilder).

Hier besteht die Indikation zur frühzeitigen invasiven Diagnostik, das heißt Herzkatheteruntersuchung mit Koronarangiographie sobald wie möglich, aber maximal innerhalb 48 Stunden ab Symptombeginn.

\section{Niedriges Risiko für Progression} zum transmuralen Infarkt

Ein niedriges Risiko haben Patienten ohne rezidivierende Angina pectoris im Beobachtungszeitraum, ohne ST-Streckensenkungen oder -hebungen sondern mit T-Negativierungen, flachen T-Wellen oder normalem EKG-Befund, sowie Patienten ohne erhöhte kardiale Troponinspiegel oder anderen biochemischen Markern bei der ersten und bei wiederholten Bestimmungen.

Bei dieser Gruppe ist ein konservatives Vorgehen zunächst gerechtfertigt. Eine invasive Diagnostik

Tab. 2 Akut-Therapie des Myokardinfarkts

\begin{tabular}{|c|c|c|}
\hline Prozedere & Maßnahmen & Bemerkungen \\
\hline Medikamentöse & 1. Analgesie (Morphin), Sedation (Diazepam) & \\
\hline \multirow[t]{7}{*}{ Akuttherapie } & 2. $\mathrm{O}_{2}$-Insufflation (2-4I/min über Nasensonde) & \\
\hline & 3. Aspisol $1 \mathrm{~g}$ i.v. oder ASS $500 \mathrm{mg}$ als Kautablette & \\
\hline & $\begin{array}{l}\text { 4. Nitroglycerin 0,4-0,8 mg Spray, s.l. oder } \\
10-20 \mu \mathrm{g} / \mathrm{min} \text {. i.v. }\end{array}$ & $\begin{array}{l}\text { KI: arterielle Hypotonie (RRsys < } 100 \text { mmHg), } \\
\text { kardiogener Schock }\end{array}$ \\
\hline & $\begin{array}{l}\text { 5. Heparin } 4000 \text { I.E. i.v. Bolus, anschließend } 1000 \text { I.E./h i.v. } \\
\text { mit therapeutischer Antikoagulation }\end{array}$ & \\
\hline & 6. $\beta$-Rezeptoren-Blocker & $\begin{array}{l}\text { z.B. Metoprolol 2,5-5 mg fraktioniert i.v. oder } 50 \text { mg } \\
\text { p.o., Ausnahmen für die generelle } \beta \text {-Rezeptoren- } \\
\text { Blocker-Gabe: bekanntes Asthma bronchiale, AV-Block } \\
\text { II-III, Bradykardie < 60/min, Hypotonie }<90 \text { mmHg } \\
\text { systolisch, klinisch manifeste Herzinsuffizienz mit } \\
\text { Lungenstauung }\end{array}$ \\
\hline & 7. Fibrinolyse & $\begin{array}{l}\text { falls keine Akut-Koronarangiographie verfügbar ist: } \\
\text { Prüfung der Kontraindikationen für Fibrinolyse, dann } \\
\text { z.B. Alteplase (r-tPA, Actilyse }{ }^{\circledR} \text { ) nach GUSTO-Schema: } \\
15 \text { mg über } 1-2 \text { Minuten, dann } 50 \text { mg über } 30 \text { Minuten, } \\
\text { anschließend } 35 \text { mg über } 60 \text { Minuten }\end{array}$ \\
\hline & 8. Volumengabe beim rechtsventrikulären Infarkt & Volumengabe nach ZVD, Zielwerte: ZVD 15-18 mmHg \\
\hline \multirow[t]{2}{*}{$\begin{array}{l}\text { Interventionelle } \\
\text { Therapie }\end{array}$} & $\begin{array}{l}\text { 1. Akut-Koronarangiographie mit Rekanalisation, } \\
\text { P TCA und Stentimplantation }\end{array}$ & $\begin{array}{l}\text { bei rascher Verfügbarkeit in der Klinik oder Nähe zu } \\
\text { einem Katheterlabor empfehlenswert (tolerierbare } \\
\text { Transportverzögerung max. } 90 \text { Minuten), in diesem Fall } \\
\text { ist Akut-PTCA der Fibrinolyse vorzuziehen. }\end{array}$ \\
\hline & 2. IABP-Implantation & $\begin{array}{l}\text { Prinzip der Intraaortalen Ballonpumpe (IABP): } \\
\text { 1. Verbesserung der Koronar- und zerebralen Perfusion, } \\
\text { 2. Nachlastsenkung (funktionelle Vasodilatation) }\end{array}$ \\
\hline $\begin{array}{l}\text { Chirurgische } \\
\text { Therapie }\end{array}$ & $\begin{array}{l}\text { Notfall-ACVB-OP in ausgewählten Fällen, z.B. Haupt- } \\
\text { stammstenose, Ventrikelseptum-Ruptur oder akuter } \\
\text { Mitralinsuffizienz bei Papillarmuskelruptur }\end{array}$ & \\
\hline
\end{tabular}


erfolgt nur bei klinischem Rezidiv oder bei pathologischem Belastungstest (Belastungs-EKG, StressEcho, Myokardszintigraphie oder Kardio-MRT).

\section{Therapie beim ST-Elevations- Myokardinfarkt (STEMI)}

Die Therapie erfolgt nach folgender Strategie: 1. Supportive Therapie zur Symptomlinderung, Begrenzung der Infarktausdehnung und Prävention von Komplikationen. 2. Kausale Behandlung des koronaren Gefäßverschlusses, das heißt Reperfusionsmaßnahmen (Tab. 2).

Zunächst erhält der Patient in Anlehnung an die Pathophysiologie thrombotischer Gefäßverschluss einer epikardialen Koronararterie eine Thrombozytenaggregationshemmung in Form von ASS (Aspisol $1 \mathrm{~g}$ i.v.) und simultan eine plasmatische Gerinnungshemmung mit Heparin (4000 I.E. i.v.) als Bolus. Nitroglycerin wird symptomatisch je nach Blutdruck und Wirkung repetitiv als Spray oder intravenös als Dauerinfusion $(10-20 \mu \mathrm{g} / \mathrm{min})$ eingesetzt. Intramuskuläre Injektionen sind wegen einer möglichen Fibrinolyseoption streng kontraindiziert. Über eine Nasensonde oder Maske wird Sauerstoff (2-4 1/min) zugeführt. Eine analgetische Therapie mit Morphin (1-3 mg fraktioniert i.v.) sollte großzügig verabreicht werden. Bei fehlenden Kontraindikationen Asthma bronchiale, Bradykardie $<60 / \mathrm{min}$ und AV-Blockierungen $>$ Grad I, Hypotonie < 90 mmHg systolische oder manifeste Herzinsuffizienz - wird dann ein ß-Rezeptorenblocker eingesetzt, beispielsweise Metoprolol 2,5-5 mg fraktioniert und langsam i.v. Ziel ist eine Herzfrequenzsenkung auf $\leq 75 / \mathrm{min}$.

Vordringliches Ziel der kausalen Behandlung ist eine möglichst rasche und vollständige Reperfusion des Infarktgefäßes. Hierzu steht traditionell seit den 80er Jahren die Fibrinolysebehandlung mit Fibrinspezifischen oder auch unspezifischen Thrombolytika zur Verfügung. In jüngerer Zeit hat aber die mechanische Rekanalisation des Infarktgefäßes mit interventionellen Verfahren, das heißt die Akut-Koronarangiographie mit Rekanalisation,
PTCA und Stentimplantation (Perkutane Koronarintervention, $\mathrm{PCI}$ ) an Bedeutung gewonnen und wird aufgrund höherer primärer Erfolgsaussichten und besserer Langzeitergebnisse der Fibrinolysetherapie vorgezogen. Dies gilt insbesondere dann, wenn der Patient innerhalb eines vertretbaren Zeitraums in ein Zentrum mit invasiv-therapeutischen Möglichkeiten aufgenommen/verlegt werden kann, in diesen Fällen ist die PCI der Fibrinolyse aus prognostischer Sicht deutlich überlegen (Tab. 3).

In den übrigen Fällen muss eine Fibrinolysetherapie nach Prüfung möglicher Kontraindikationen angestrebt werden (Tab. 4). In der Klinik soll die Zeit bis zum Beginn einer Fibrinolyse möglichst unter $30 \mathrm{Mi}$ nuten liegen („door-to-needle-time“).

Erfahrungsgemäß werden ältere Infarktpatienten häufig ohne medizinische Rationale nur unzureichend aggressiv diagnostiziert und therapiert. Aufgrund des Alters wird bisweilen eine adäquate Abklärung und Behandlung vorenthalten, welche einzig eine signifikante Prognoseverbesserung durch Erhalt des linksventrikulären Myokards und Verhinderung eines kardiogenen Schocks bewirken kann. Intensivmedizinische Überwachung und frühzeitige Erfassung und Therapie von Komplikationen bewirken unabhängig von Reperfusionsstrategien eine erhebliche Mortalitätssenkung. In einzelnen Fällen wird durch frühzeitige invasive Diagnostik die Indikation zur notfallmäßigen operativen Therapie gestellt, wie bei kritischer Stenose des Hauptstamms der linken

\section{Tab. 3 Empfehlungen der Europäischen Gesellschaft für Kardiologie zur primären $\mathrm{PCl}$ bei akutem Myokardinfarkt mit ST-Elevation}

$\mathrm{PCl}$ indiziert:

- Wenn die PCl von einem erfahrenen Team und in einem qualifizierten Zentrum innerhalb von 90 min. ab dem ersten Arztkontakt durchgeführt werden kann.

- Wenn die Thrombolyse kontraindiziert ist.

- Bei Patienten mit kardiogenem Schock.

Koronararterie sowie bei Infarktassoziierten Komplikationen wie Ventrikelseptum-Ruptur und akuter Mitralklappeninsuffizienz durch Papillarmuskelruptur. Die perioperative Mortalität ist in diesen Situationen natürlich deutlich erhöht.

\section{Therapie beim Non-ST- Elevations-Infarkt (NSTEMI) und instabiler Angina pectoris (iAP)}

Die Therapie erfolgt nach folgender Strategie: 1. Risikoklassifizierung des individuellen Patienten, 2. intensivierte antithrombotische Therapie und 3. Akutintervention (PCI) bei Hochrisiko-Patienten.

Auch hier erfolgt die Risikostratifizierung in zwei Gruppen.

\section{Patienten mit hohem Risiko für Progression zum transmuralen Infarkt}

Die intensivierte antithrombotische Therapie besteht in der Gabe von ASS in Kombination mit einem Heparin-Präparat, vorzugsweise einem niedermolekularen Heparin (LMWH), einem ADP-Rezeptorantagonisten (Tripeltherapie) sowie ge-

\section{Tab. 4 Empfehlungen der Europäischen Gesellschaft für Kardiologie zur Thrombolyse}

- Wenn die Voraussetzungen für eine prompte PCI in einem qualifizierten Zentrum mit einem erfahrenen Team nicht erfüllt sind.

- Wenn der Symptombeginn weniger als 1-2 Stunden zurückliegt (hohe Erfolgschancen der Thrombolyse).

- Wenn der Symptombeginn >4 Stunden zurückliegt, sollten fibrinspezifische Thrombolytika (Alteplase, Tenecteplase) bevorzugt werden.

- Unfraktioniertes Heparin soll über 24-48 Stunden in angepasster Dosis (max. $4000 \mathrm{E}$ Bolus gefolgt von $12 \mathrm{E} / \mathrm{kg} / \mathrm{h}$ ) infundiert werden.

- Die Kombination von niedermolekularen Heparinen mit Fibrinolyse erhöht die Gefahr intrazerebraler Blutungen und ist derzeit vor allem bei älteren Patienten (> 75 Jahre) nicht zu empfehlen. 
gebenenfalls einem GlykoproteinIIb/IIIa-Antagonisten (QuadrupelTherapie).

ASS: durch Hemmung der Cyclooxygenase wird die Synthese von Thromboxan A2 gehemmt, einem potenten Agonisten der Aggregation. Durch ASS allein wird die Thrombozytenaggregation aber nicht vollständig blockiert, sondern lediglich zu etwa 20\%. Dennoch ist die protektive Wirkung von ASS (75-325 mg/die) beim akuten Koronarsyndrom in zahlreichen Studien belegt, ASS ist auch einzig im Langzeitverlauf > 12 Monate zur Senkung des kardiovaskulären Risikos gesichert wirksam. Eine zusätzliche Thrombozytenaggregationshemmung wird durch Gabe von Clopidogrel - einem Thienopyridin mit Hemmung des ADP-Rezeptors auf der Thrombozytenoberfläche - erreicht (Abb. 4). Durch die Kombinationsbehandlung wird eine intensivere Wirkung erreicht, Clopidogrel alleine bewirkt wiederum eine Aggregationshemmung von nur zirka 20\%. Die Wirksamkeit von Clopidogrel zur Sekundärprophylaxe beziehungsweise bei akutem Koronarsyndrom wurde unter anderem in der CAPRIE- und der CURE-Studie untersucht. Clopidogrel war dabei ASS alleine überlegen, es konnte ein synergistischer Effekt der Kombination über zwölf Monate Therapiedauer nachgewiesen werden. Aufgrund eines verzögerten Wirkungseintritts (1-2 Tage) wird Clopidogrel üblicherweise mit einer so genannten loading-dose von $300 \mathrm{mg}$, gefolgt von $75 \mathrm{mg} /$ die verabreicht. Neuere Daten zeigen einen erheblich rascheren Wirkungseintritt bereits nach wenigen Stunden bei einer loading dose von 600 mg ohne signifikant höhere Nebenwirkungsrate. Eine nahezu vollständige Thrombozytenaggregationshemmung (> 80\%) wird durch Blockade der gemeinsamen Endstrecke der Thromobzytenaktivierung, des Glykoprotein-IIb/IIIa-Rezeptors erreicht, der Thrombozyten mit Fibrinogen vernetzt. Gegenwärtig stehen drei Substanzen zur intravenösen Applikation zur Verfügung, die Resultate klinischer Studien mit oralen Präparaten waren enttäuschend. Abciximab (ReoPro ${ }^{\circledR}$ ), Tirofiban (Aggra- stat $^{\circledR}$ ) und Eptifibatid (Integrilin ${ }^{\circledR}$ ) unterscheiden sich im Wesentlichen im Hinblick auf pharmakokinetische Eigenschaften und Wirkungsdauer. Allen Substanzen ist gemeinsam, dass sie einen raschen Wirkungseintritt nach wenigen Minuten aufweisen und durch kompetitive Verdrängung von Fibrinogen offenbar auch Thromben aufzulösen vermögen. In klinischen Studien wurden alle drei Substanzen intensiv bei Patienten mit akuten Koronarsyndromen untersucht. Es zeigte sich in Kombination mit ASS jeweils eine signifikante Reduktion der Endpunkte Tod und Myokardinfarkt insbesondere bei denjenigen Patienten, die sich einer perkutanen Koronarintervention unterzogen $(\mathrm{PCI})$, bei Patienten mit erhöhten Troponin-Spiegeln sowie bei Diabetikern. Das heißt, Patienten mit hohem Risiko oder einer frühen Koronarintervention profitieren ausgeprägter von einer hochaktiven Thrombozytenaggregationshemmung. Die Therapie mit einem GP-IIb/IIIa-Hemmer sollte daher früh eingeleitet werden, zum Beispiel nach erfolgter Risikostratifizierung und zum Transport des Patienten in ein Zentrum mit Interventionsmöglichkeiten, in einzelnen Fällen ist der Beginn der Behandlung während der Herzkatheteruntersuchung ausreichend. Ob im individuellen Fall eine Tripel- oder Quadrupeltherapie indiziert ist, wird durch den Bedarf eines raschen Wirkungseintritts, beispielsweise bei komplexer Koronarintervention und visuel- lem Thrombennachweis, begründet. Evidenzbasierte Daten zur Quadrupeltherapie im Vergleich zur Tripeltherapie der Thrombozyten-Aggregationshemmung sind bisher nicht vorhanden.

Die Thrombozyten-Aggregationshemmung wird durch eine plasmatische Gerinnungshemmung ergänzt. Für unfraktioniertes (UFH) und für niedermolekulares Heparin (LMWH) ist eine prophylaktische Wirkung mit Senkung der Endpunkte Tod oder Myokardinfarkt nachgewiesen. Der Vergleich der einzelnen niedermolekularen Heparine ergab in der ESSENCE- und der TIMI-11B-Studie einen Vorteil für Enoxaparin im Vergleich zu unfraktioniertem Heparin, die übrigen LMWH sind gleich wirksam wie unfraktioniertes Heparin.

Fibrinolytika haben bei der Therapie des akuten Koronarsyndroms ohne ST-Hebung keinen Stellenwert. Aus kleineren Studien ist abzuleiten, dass sie die Prognose bei iAP und NSTEMI verschlechtern.

Nach Risikoklassifizierung und Einleitung einer intensivierten antithrombotischen Therapie folgt die frühzeitige invasive Diagnostik mittels Herzkatheteruntersuchung und Koronarangiographie. Nach diagnostischer Angiographie können vorhandene Koronarstenosen und flusslimitierende aufgebrochene Plaques einer sofortigen interventionellen Therapie mittels PTCA in der Regel mit Stentimplantation (PCI) zugeführt werden. In ausgewählten

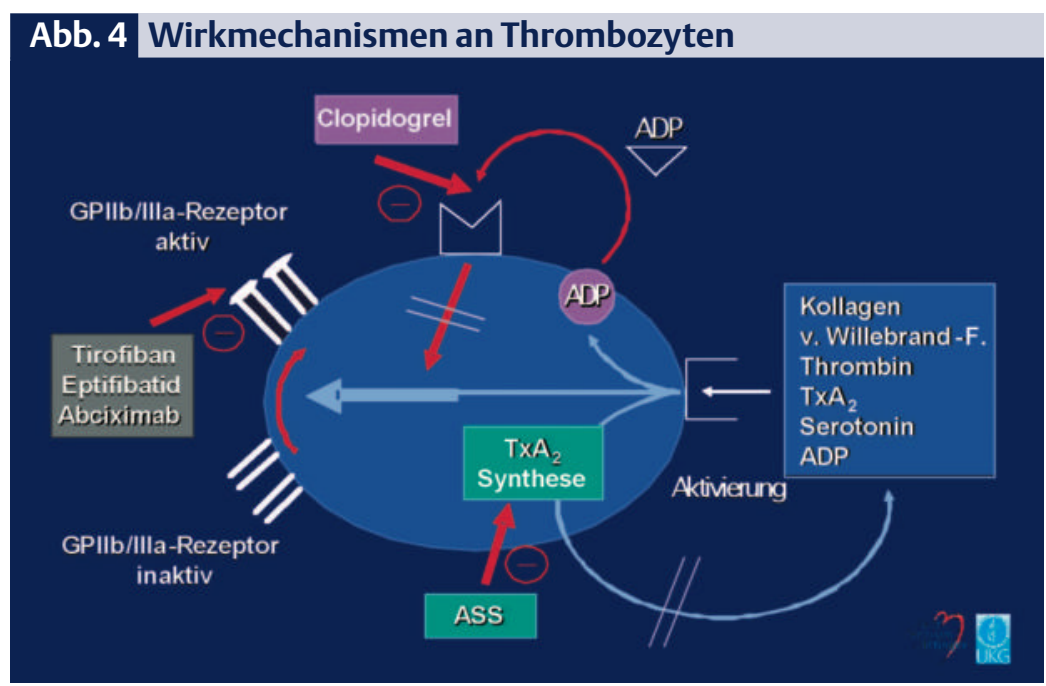


Fällen mit interventionell nicht behandelbaren Läsionen, schwerer koronarer Drei-Gefäßerkrankung und bei relevanter Hauptstammstenose der linken Kranzarterie kann die Indikation zur dringlichen aortokoronaren Bypassoperation gestellt werden. Dieses aggressive Vorgehen hat sich nach Ergebnissen mehrerer Studien gegenüber einer konservativen Therapie mit medikamentöser Stabilisierung des akuten Koronarsyndroms bewährt, auch unter optimaler medikamentöser Therapie einschließlich GP-IIb/IIIaInhibitoren und LMWH profitieren die Patienten dennoch von einer früh-invasiven Strategie (z.B. FRISC II, TACTICS-TIMI 18-Studie). Das heißt, ein GP-IIb/IIIa-Inhibitor alleine kann die konsequente Revaskularisationsmaßnahme nicht ersetzen, beide Verfahren ergänzen sich jedoch in sinnvoller Weise.

\section{Patienten mit niedrigem Risiko} für Progression zum transmuralen Infarkt

Die antithrombotische Therapie wird mit ASS $100 \mathrm{mg} /$ die und Clopidogrel (Initialdosis 300-600 mg, dann $75 \mathrm{mg} /$ die) eingeleitet, eine plasmatische Gerinnungshemmung ist in der Regel nicht erforderlich und kann nach der initialen Beobachtungsphase abgesetzt werden. Der Patient erhält zur Herzfrequenzund damit Sauerstoffbedarfsenkung einen kardioselektiven $\beta$-Blocker und bei Bedarf zur Symptomlinderung und -prophylaxe Nitrate sowie bei hypertensiven Blutdruckwerten Calciumantagonisten (Cave: unretardiertes Nifedipin). Daneben werden Maßnahmen zur Sekundärprävention eingeleitet (Statine, Nikotinkarenz, RR-Einstellung).

Der Patient wird anschließend einer Belastungsuntersuchung mittels Belastungs-EKG, Myokardszintigraphie, Stressecho oder KardioMRT zugeführt und das weitere Vorgehen in Abhängigkeit von den Resultaten festgelegt. Bei durchgehend normalem Ruhe-EKG, negativem Belastungstest und negativen biochemischen Markern können die Symptome des Patienten möglicherweise durch nicht-kardiale Befunde erklärt werden und Untersuchungen

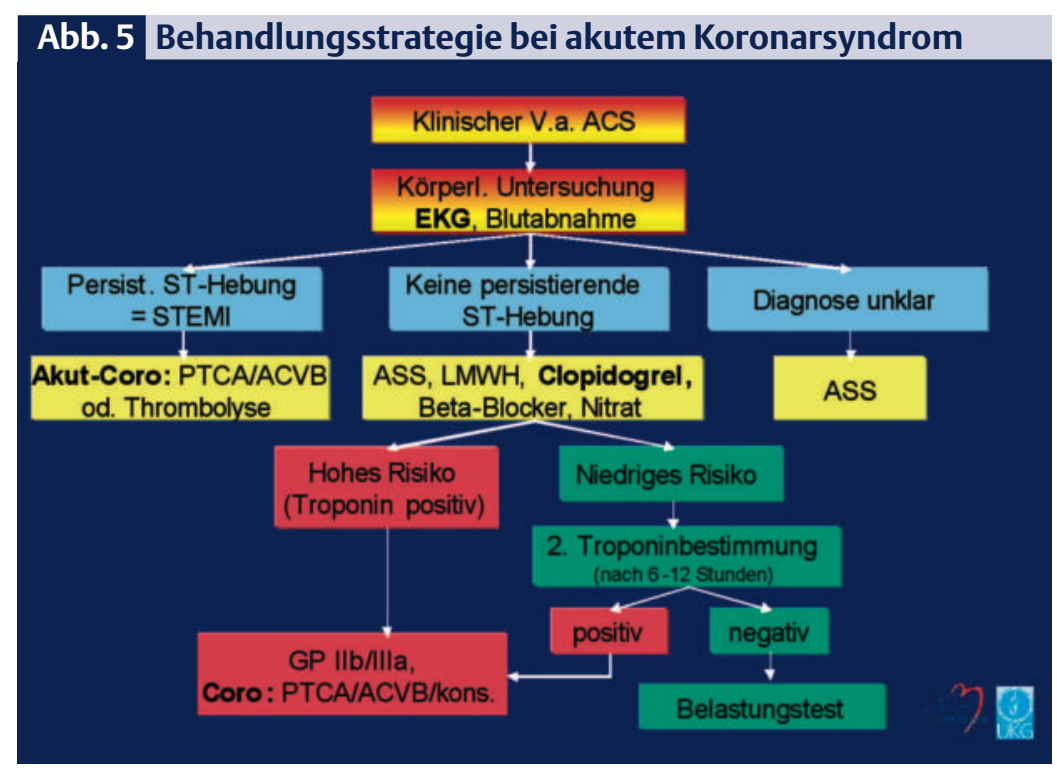

anderer Organsysteme werden erforderlich. Das Risiko für ein kardiales Ereignis ist bei diesen Patienten sehr gering, die weiteren Untersuchungen können daher in der Regel zu einem späteren Zeitpunkt ambulant erfolgen. Patienten mit Zeichen der belastungsabhängigen Myokardischämie, das heißt positiven Belastungstests, werden einer elektiven invasiven Koronardiagnostik zugeführt.

Den zusammenfassenden Therapiealgorithmus bei akutem Koronarsyndrom zeigt Abb. 5.

\section{Langzeittherapie bei Patienten mit akutem Koronarsyndrom}

Als Dauertherapie nach akutem Koronarsyndrom wird ASS 100 mg und Clopidogrel 75 mg für mindestens 9-12 Monate verordnet. Als weitere Maßnahmen bei gesicherter koronarer Herzkrankheit wird lebenslang ein ACE-Hemmer, vorzugsweise Ramipril 5-10 mg sowie ein Statin (Ziel: LDL-Cholesterin $<100 \mathrm{mg} / \mathrm{dl}$ ) und ein $\beta$-Blocker verabreicht.

\section{Summary}

According to the new WHO definition, a myocardial infarction presents when, in patients with unstable angina pectoris, biochemical signs/markers of myocardial damage are detectable, that is, elevated CK-MB or car- diac troponin levels (troponin T or I) are present.

Current treatment is based on a knowledge of the common pathophysiology of acute coronary syndromes, and is stage-specific. Large epidemiological studies have shown that the prognosis is unfavourable, not only in ST-segment elevation infarctions, but also in acute coronary syndrome without persistent ST-segment elevation. This justifies an aggressive therapeutic approach analogous to that applicable in myocardial infarction with ST-segment elevation.

\section{Literatur}

1. Bertrand ME, Simoons ML, Fox KAA et al. Management of acute coronary syndromes in patients presenting without persistent ST-segment elevation. Eur Heart J 2002; 23: 1809

2. Braunwald E, Antman EM, Beasley JW et al. ACC/AHA 2002 guideline update for the management of patients with unstable angina and non-ST-segment elevation myocardial infarction-2002: summary article. Circulation 2002; 106: 1893

\section{Anschrift des Verfassers}

PD Dr. med. Hans-Peter Hermann

Herzzentrum Göttingen

Abteilung Kardiologie und Pneumologie

Georg-August-Universität Göttingen

Robert-Koch-Straße 40

37075 Göttingen

Fax: 05 51/3989 18

E-Mail: phermann@med.uni-goettingen.de 